\title{
Multiphoton cross sections of conductive electrons stimulated bremsstrahlung in doped bilayer graphene
}

\author{
A.G. Ghazaryar* and Kh.V. Sedrakian \\ Centre of Strong Fields, Yerevan State University, 1 A. Manukian, Yerevan 0025, Armenia
}

(Dated: July 11, 2019)

\begin{abstract}
The quantum theory of multiphoton stimulated bremsstrahlung of charged carriers on an arbitrary electrostatic potential of impurity ion in doped bilayer graphene at the presence of coherent electromagnetic radiation is developed. A terahertz wave field is considered exactly, while the electrostatic potential of doped ions as a perturbation. The essentially nonlinear response of bilayer graphene to a pump wave and significant differences from the case of a single layer graphene are shown, which can be associated to nonlinear parabolic dispersion. The latter opens new way to manipulate with the electronic transport properties of conductive electrons of bilayer graphene by coherent radiation field of terahertz or near-infrared frequencies.
\end{abstract}

PACS numbers: $42.50 . \mathrm{Hz}, 34.80 . \mathrm{Qb}, 32.80 . \mathrm{Wr}, 31.15 .-\mathrm{p}$

\section{INTRODUCTION}

More than twenty years single layer graphene (SG) [1, 2] has attracted the giant interest for its unusual electronic transport and the characteristics of relativistic charge carriers behaving like massless chiral Dirac fermions [3 14]. Bilayer graphene (BG) are consisted of two graphene $\mathrm{AB}$ stacked single layers. If the SG graphene band structure has a linear dispersion, BG has a quadratic dispersion [15 19] in the low-energy mode, which makes it similar to 2D semiconductor systems [20], except for the absence of an energy gap. The BG is useful for both technological and fundamental applications [15 19], 21 24]. In particular, the BG is of current interest because of a tunable bandgap [22], 25 27], and extraordinary quantum Hall physics with an eight-fold degenerate zero energy Landau level [18], and anomalous disorder and charge-carrier scattering [28-30]. Moreover, BG is of interest by considerably richer properties at multiphoton resonant excitations and high harmonic generation [11], 31], 32]. However, little is known about the effective absorption of coherent electromagnetic (EM) radiation via the multiphoton stimulated bremsstrahlung (SB) process [5] on charged impurity ions in BG. Among the induced by external radiation field processes SB is one of the first stimulated effects at laser-matter interaction, revealed after the invention of lasers [33], 34]. The SB process is the main mechanism of energy exchange between charged particles and a plane monochromatic wave in plasma-like medium for real absorption-emission processes.

As it is known, the interaction of electron with the EM wave in initially nonrelativistic plasma media and the photon energy $\hbar \omega>T_{e}$ is described by dimensionless intensity parameter of intensity $\chi_{0}=e E_{0} / \omega \sqrt{m_{e} \hbar \omega}$ [5] $\left(E_{0}\right.$ is the wave field strength amplitude, $\omega$ is the frequency of the wave, $e$ and $m_{e}$ are the elementary charge and mass, $T_{e}$ is the electrons temperature). The $\chi_{0}$ is the ratio of the amplitude of the momentum given by the wave field to momentum at the one-photon absorption. Hence the intensity of the wave expressed by the parameter $\chi_{0}$ can be estimated as $I_{\chi_{0}}=\chi_{0}^{2} \times 1.74 \times 10^{12} \mathrm{Wcm}^{-2}(\hbar \omega / \mathrm{eV})^{3}[\underline{8}]$. Multiphoton effects become essential at $\chi_{0} \sim 1$, which for terahertz photons $\hbar \omega \sim 0.01 \mathrm{eV}$ corresponds to intensity $I_{\chi_{0}}=1.74 \times 10^{6} \mathrm{Wcm}^{-2}$. Meanwhile, in BG for intraband transitions the wave-particle interaction at the photon energies $\hbar \omega>\mathcal{E}_{L}$ [11] characterizes by known dimensionless intensity parameter $\chi=\chi_{0}\left(m_{e} \rightarrow m_{*}\right)\left(m_{*}\right.$ is an effective mass of chiral particle, $\mathcal{E}_{L} \simeq 1 \mathrm{meV}$ is the Lifshitz energy). Depending on the value of the parameter $\chi$, three regimes of the wave-particle interaction can take place: $\chi \ll 1$ corresponds to one-photon interaction, $\chi \gg 1$, which is the static field limit or so called Schwinger regime [35], and $\chi \succsim 1-$ corresponds the multiphoton interaction [6]. The wave intensity for the given $\chi$ can be expressed as $I_{\chi}=\chi^{2} \times 6 \times 10^{10} \mathrm{Wcm}^{-2}(\hbar \omega / \mathrm{eV})^{3}$ [1]. Comparison of this intensity threshold with the analogous one for the plasma electrons shows the substantial difference $I_{\chi_{0}=1} / I_{\chi=1} \sim 30$ between the values of these thresholds (instead of the light speed here stands up the Fermi velocity -much less than the light speed in vacuum). Thus, for realization of multiphoton SB in BG one can expect $\sim 30$ times smaller intensities than for SB in atoms [7], [8], 33, 36, 37]. In addition, the considered in the present paper pump wave photon energy range of interest lies in the terahertz or near infrared domain, where the high-power terahertz or near-infrared generators and frequency multipliers are of special

*Electronic address: amarkos@ysu.am 
interest. In general, the role of terahertz or near infrared radiation for the study of nonlinear phenomena in condensed matter physics is important.

Regarding the investigation of electrons elastic scattering on impurity ions in intrinsic SG, it has been investigated mainly within the framework of perturbation theory by electrostatic potential (see, e.g., 38 44]). The SB process in graphene at moderate intensities of stimulated radiation, in case of its linear absorption by electrons (or holes), there are extensive investigations carried out in the scope of the linear theory (see, e.g. [45-49]). Multiphoton cross-sections for SB of conductive electrons in intrinsic SG have been obtained in the Born approximation over the scattering potential in the presence of an external EM radiation (see, [50], 51]). The transport properties of the BG are studied theoretically within a self-consistent Born approximation in the papers [16]. The transport and elastic scattering times on impurities in SG and BG are investigated experimentally and comparison with theoretical predictions have been made in 52 57].

In the present paper the formerly developed [50] quantum mechanical consideration of nonlinear stimulated scattering of charged carriers on the Coulomb field of impurity ions in SG in the presence of an external strong coherent EM radiation was extended to doped BG. The selected frequency range of terahertz radiation excludes the valence electrons excitations at high Fermi energies. The interaction of electrons with the scattering potential is considered in the Born approximation. The analytic formulas in the case of screened Coulomb potential have been analyzed numerically for actual parameters of interaction system in the strong radiation field. The results of our investigations show that in the terahertz domain of wave frequencies one can achieve efficient rates of absorption of pump waves already at moderate intensities by this mechanism.

In Sec. II the multiphoton rates and total cross-sections of SB process of conductive electrons on the charged impurity ions in BG have been obtained. In Sec. III the analytic formulas in case of screened Coulomb potential have been analyzed numerically and the results for the partial differential cross-sections of SB, as well as the angular dependence of scattered electrons on the incident radiation intensity are received. Conclusions are given in Sec. IV.

\section{MULTIPHOTON TRANSITION AMPLITUDES AND CROSS-SECTIONS OF 2D DIRAC FERMIONS STIMULATED BREMSSTRAHLUNG IN DOPED BILAYER GRAPHENE}

In the following we will develop the scattering theory for the $2 \mathrm{D}$ chiral fermions on arbitrary electrostatic potential $U(\mathbf{r})$ of an impurity ion in doped BG at the presence of external EM wave of moderate intensities, in analogous with intrinsic SG [50]. Using the scattering Green function formalism in the Born approximation by potential $U(\mathbf{r})$ we will obtain the SB rates. Note, that the pioneer treatment of nonrelativistic SB in the Born approximation has been carried out analytically in [33]. Then this approach has been extended to the relativistic domain [36], [37]. In accordance with the quantum theory, the transition amplitude of SB process in the coherent EM radiation field from the state with the canonical momentum $\mathbf{p}_{0}\left(p_{x}, p_{y}\right)$ to the state with momentum $\mathbf{p}\left(p_{x}, p_{y}\right)$ in graphene plane $(X, Y)$, can be written as:

$$
T_{\mathbf{p p}_{0}}=-\frac{i}{\hbar} \int \Psi_{\mathbf{p}}^{+}(\mathbf{r}, t) U(\mathbf{r}) \Psi_{\mathbf{p}_{0}}(\mathbf{r}, t) d t d \mathbf{r}
$$

where bispinor function $\Psi^{+}$is the complex conjugation of $\Psi$.

We use an even simpler model for BG which neglects both the electron-hole asymmetry and the trigonal warping. To exclude the valence electrons excitations at high Fermi energies in graphene, we will assume for EM wave to be a terahertz radiation. The $\mathrm{BG}$ system is still chiral due to the $A / B$ sublattice symmetry giving rise to the conserved pseudospin quantum index. The detailed chiral 4-component wavefunction for the bilayer case including both layer and sublattice degrees of freedom can be found in the original literature in [15], 223], [25], [28], [29]. In accordance with the nonlinear quantum theory of BG, in the mentioned case with energy window $0.002 \mathrm{eV} \lesssim \varepsilon \lesssim 0.1 \mathrm{eV}$ in the vicinity of Dirac points $K_{\zeta}$ (valley quantum number $\zeta= \pm 1$ ) in the Brillouin zone, the fermion particle wave function $\Psi_{\mathbf{p}}(\mathbf{r}, t)$ in the strong EM wave field may be presented in the form:

$$
\Psi_{\mathbf{p}}(\mathbf{r}, t)=\frac{1}{\sqrt{S}} F_{\mathbf{p} \sigma} e^{\frac{i}{\hbar} \mathbf{p r}-i \Omega(\mathbf{p}, t)} .
$$

Here parameter $S$ is the quantization area-graphene layer surface area,

$$
F_{\mathbf{p} \sigma}=\frac{1}{\sqrt{2}}\left(\begin{array}{c}
e^{i 2 \zeta \vartheta} \\
\sigma
\end{array}\right)
$$




$$
\Omega(\mathbf{p}, t)=\frac{1}{2 m_{*} \hbar} \int\left[\left(p_{x}+\frac{e}{c} A_{x}\right)^{2}+p_{y}^{2}\right] d t,
$$

are the spinor wave function $F_{\mathbf{p} \sigma}$ and the temporal phase $\Omega(\mathbf{p}, t)$. The function $\vartheta\left(\mathbf{p}+\frac{e}{c} \mathbf{A}(t)\right)$, given in the form:

$$
\vartheta\left(\mathbf{p}+\frac{e}{c} \mathbf{A}(t)\right)=\arctan \left(p_{y} /\left(p_{x}+\frac{e}{c} A_{x}\right)\right)
$$

defines the angle between the vectors of particle kinematic momentum $\mathbf{p}=\mathbf{p}\left(p_{x}+\frac{e}{c} A_{x}, p_{y}\right)$ in the EM field and the wave vector potential $\mathbf{A}(t)=-c \int_{\Omega}^{t} \mathbf{E}\left(t^{\prime}\right) d t^{\prime}$ (unit vector $\widehat{\mathbf{e}}$ is directed along the axis $O X$ ). The BG has a low-energy dispersion which is approximated [18], [29] by massive valence and conduction bands without gap (in opposite to zeromass bands in SG). The quasiparticle energy $\varepsilon(p)$ is defined by $\varepsilon(p)=\sigma\left(p_{x}^{2}+p_{y}^{2}\right) / 2 m_{*}$, where $\sigma= \pm 1$ correspond to the conduction/valence bands; $m_{*}=\gamma_{1} /\left(2 \mathrm{v}_{F}^{2}\right), \gamma_{1} \simeq 0.39 \mathrm{eV}$ is the interlayer tunneling amplitude, $\mathrm{v}_{F}$ is the intrinsic SG Fermi velocity. This parabolic dispersion applies only for small values of $p$ satisfying $p / \hbar \ll \gamma_{1} /\left(\hbar \mathrm{v}_{F}\right)$ and for carrier densities (or equivalently, the impurity densities) smaller than $5 \times 10^{12} \mathrm{~cm}^{-2}$, respectively. In the opposite limit, $p / \hbar \gg \gamma_{1} /\left(\hbar \mathbf{v}_{F}\right)$ and for the carrier densities larger than the last, we get a linear band dispersion $\varepsilon(p)=\sigma \hbar p \mathbf{v}_{F}$, just as in the intrinsic SG case [58]. For the actual parameters the effective mass for BG is $m_{*} \approx(0.033 \div 0.05) m_{e}$, with free electron mass $m_{e}$.

The spin and the valley quantum numbers are conserved. There is no degeneracy upon the valley quantum number $\zeta$ for the issue considered. However, since there are no intervalley transitions, the valley index can be considered as a parameter.

The wave will be applied in the perpendicular direction to the BG sheet $(X Y)$ to exclude the effect of the magnetic field. For the simplest the plane quasimonochromatic EM wave of frequency $\omega$ is taken to be linearly polarized along the $X$ axis: $E(t)=E_{0} \cos \omega t$. It is clear that at such small Fermi velocities of scattered particles the plane monochromatic wave will turn into the uniform periodic electric field of frequency $\omega=2 \pi / T$. Similar calculations for a wave linearly polarized along the $X$ axis show qualitatively the same picture.

The known formalism of the time dependent scattering theory can be applied to obtain the multiphoton $(\chi \succsim 1)$ SB process transition amplitude $T_{\mathbf{p p}_{0}}$ between the unperturbed states $\Psi_{\mathbf{p}}$ and $\Psi_{\mathbf{p}_{0}}$. Thus, we can write for transition amplitude the following ratio:

$$
T_{\mathbf{p p}_{0}}=-\frac{i}{\hbar} \int F_{\mathbf{p}}^{+}(t) U(\mathbf{r}) F_{\mathbf{p}_{0}}(t) \exp \left(\frac{i}{\hbar}\left(\mathbf{p}-\mathbf{p}_{0}\right) \mathbf{r}\right) d t d \mathbf{r},
$$

which can be expressed in the form:

$$
T_{\mathbf{p p}_{0}}=-\frac{i}{\hbar} \int F_{\mathbf{p}}^{+}(t) \widetilde{U}\left(\frac{\mathbf{p}-\mathbf{p}_{0}}{\hbar}\right) F_{\mathbf{p}_{0}}(t) d t
$$

where

$$
\widetilde{U}\left(\frac{\mathbf{p}-\mathbf{p}_{0}}{\hbar}\right)=\int \exp \left(\frac{i}{\hbar}\left(\mathbf{p}-\mathbf{p}_{0}\right) \mathbf{r}\right) U(\mathbf{r}) d \mathbf{r}
$$

is the Fourier transform of the scattering potential. Taking into account the relation (7) for the impurity potential of the arbitrary form $\widetilde{U}$, we will have for $T_{\mathbf{p p}_{0}}$ the relation:

$$
\begin{gathered}
T_{\mathbf{p p}_{0}}=-\frac{i}{\hbar} \frac{1}{2 S} \widetilde{U}\left[\frac{\mathbf{p}-\mathbf{p}_{0}}{\hbar}\right] \\
\times \int\left(1+e^{i 2 \zeta\left[\vartheta\left(\mathbf{p}_{0}+\frac{e}{c} \mathbf{A}(\tau)\right)-\vartheta\left(\mathbf{p}+\frac{e}{c} \mathbf{A}(t)\right)\right]}\right) \\
\times e^{-\frac{i}{\hbar} \frac{1}{2 m_{*}} \int_{0}^{\tau}\left[\left(p_{x}+\frac{e}{c} A_{x}\right)^{2}+p_{y}^{2}-\left(p_{0 x}+\frac{e}{c} A_{x}\right)^{2}+p_{0 y}^{2}\right] d t} d \tau .
\end{gathered}
$$

Using the Eq. (9), the impurity ion potential can be represented in the following formula:

$$
T_{\mathbf{p p}_{0}}=\int B(\tau) e^{-\frac{i}{\hbar}\left(\mathcal{E}-\mathcal{E}_{0}\right) \tau} d \tau
$$


where the periodic function $B(\tau)$ of time defines as

$$
\begin{gathered}
B(\tau)=-\frac{i}{\hbar} \frac{1}{2 S} \widetilde{U}\left[\frac{\mathbf{p}-\mathbf{p}_{0}}{\hbar}\right] \\
\times\left(1+e^{i 2 \zeta\left[\vartheta\left(\mathbf{p}_{0}+\frac{e}{c} \mathbf{A}(t)\right)-\vartheta\left(\mathbf{p}+\frac{e}{c} \mathbf{A}(t)\right)\right]}\right) \\
\times e^{-\frac{i}{\hbar} \int_{0}^{\tau}\left[\left(\frac{1}{2 m_{*}}\left(p_{x}+\frac{e}{c} A_{x}\right)^{2}+\frac{p_{y}^{2}}{2 m_{*}}-\mathcal{E}\right)-\left(\frac{1}{2 m_{*}}\left(p_{0 x}+\frac{e}{c} A_{x}\right)^{2}+\frac{p_{0 y}^{2}}{2 m_{*}}-\mathcal{E}_{0}\right)\right] d t},
\end{gathered}
$$

and the time-depended modules of the "quasienergies" $\mathcal{E}_{0}, \mathcal{E}$ are:

$$
\begin{gathered}
\mathcal{E}_{0}=\frac{\omega}{4 \pi m_{*}} \int_{0}^{\frac{2 \pi}{\omega}}\left[\left(p_{0 x}+\frac{e}{c} A_{x}(t)\right)^{2}+p_{0 y}^{2}\right] d t, \\
\mathcal{E}=\frac{\omega}{4 \pi m_{*}} \int_{0}^{\frac{2 \pi}{\omega}}\left[\left(p_{x}+\frac{e}{c} A_{x}(t)\right)^{2}+p_{y}^{2}\right] d t,
\end{gathered}
$$

(with corresponding "quasimoumentums" $\mathbf{P}_{0}\left\{p_{0 x}+\frac{e}{c} A_{x}(t), p_{0 y}\right\}, \mathbf{P}\left\{p_{x}+\frac{e}{c} A_{x}(t), p_{y}\right\}$ ). Further we made the Fourier transformation of the function $B(t)$ over $t$ by the relations:

$$
\begin{gathered}
B(t)=\sum_{n=-\infty}^{\infty} \widetilde{B}_{n} \exp (-i n \omega t), \\
\widetilde{B}_{n}=\frac{\omega}{2 \pi} \int_{0}^{2 \pi / \omega} B(t) \exp (i n \omega t) d t .
\end{gathered}
$$

Thus, carried out the integration over $t$ in the formula (10), we have obtained for the transition amplitude $T_{\mathbf{p p}_{0}}$ :

$$
T_{\mathbf{p p}_{0}}=2 \pi \hbar \widetilde{B}_{n} \delta\left(\mathcal{E}-\mathcal{E}_{0}-n \hbar \omega\right) .
$$

The differential probability $d W_{\mathbf{p p}_{0}}$ of SB process in strong pump wave field per unit time, from the electron or hole state with two-dimensional momentum $\mathbf{p}_{0}$ to a state with momentum $\mathbf{p}$ in the phase space $g_{v} g_{s} S d \mathbf{P} /(2 \pi \hbar)^{2}$ [58] in the Born approximation may be described by the formula:

$$
d W_{\mathbf{p p}_{0}}=\lim _{t \rightarrow \infty} \frac{1}{t}\left|T_{\mathbf{p p}_{0}}\right|^{2} g_{v} g_{s} S \frac{d \theta P d P}{(2 \pi \hbar)^{2}}
$$

where $d \theta$ is the differential scattering angle. Here the degeneracy factor is $g_{v} g_{s}=4$ due to valley and spin degeneracies.

Dividing the differential probability $W_{\mathbf{p p}_{0}}$ (14) of SB by initial flux density $\left|\mathbf{P}_{0}\right| / m_{*}$ and integrating over $d P$, we can obtain the multiphoton differential cross-section $d \Lambda / d \theta$ of SB for quasiparticles in doped BG:

$$
\frac{d \Lambda}{d \theta}=\sum_{n=-n_{\max }}^{\infty} \frac{d \Lambda^{(n)}}{d \theta}
$$

where

$$
\frac{d \Lambda^{(n)}}{d \theta}=\left.\frac{2 m_{*}^{2}}{\pi \hbar^{2}}\left|\frac{\mathbf{P}}{\mathbf{P}_{0}}\right|\left|\widetilde{B}_{n}\right|^{2}\right|_{P^{2}=P_{0}^{2}+2 m_{*} \hbar \omega n}
$$

is the partial differential cross-section of $n$-photon SB with maximum number of emitted photons $n_{\max }$. The total multiphoton scattering cross-section $d \Lambda / d \theta$ will be obtained making summation over the photon numbers in the formula for differential partial cross-sections $d \Lambda^{(n)} / d \theta$ (16), which may be expressed by the following form: 


$$
\begin{gathered}
\frac{d \Lambda^{(n)}}{d \theta}=\left|\widetilde{U}\left[\frac{\mathbf{p}-\mathbf{p}_{0}}{\hbar}\right]\right|^{2} \\
\times \mid \int_{0}^{T} d\left(\frac{t}{T}\right)\left(1+e^{i 2 \zeta\left[\vartheta\left(\mathbf{p}_{0}+\frac{e}{c} \mathbf{A}(t)\right)-\vartheta\left(\mathbf{p}+\frac{e}{c} \mathbf{A}(t)\right)\right]}\right) \exp (i n \omega t) \\
\times e^{-\left.\frac{i}{\hbar} \int_{0}^{\tau}\left[\left(\frac{1}{2 m_{*}}\left(p_{x}+\frac{e}{c} A_{x}\right)^{2}+\frac{p_{y}^{2}}{2 m_{*}}-\mathcal{E}\right)-\left(\frac{1}{2 m_{*}}\left(p_{0 x}+\frac{e}{c} A_{x}\right)^{2}+\frac{p_{0 y}^{2}}{2 m_{*}}-\mathcal{E}_{0}\right)\right] d t^{\prime}\right|^{2}} \\
\times \delta\left(\frac{P^{2}}{2 m_{*}}-\frac{P_{0}^{2}}{2 m_{*}}-\hbar \omega n\right) \frac{2 m_{*} P d P}{P_{0} \pi \hbar^{3}} .
\end{gathered}
$$

We produce the expansion in Eq. (18) into a Taylor series, keeping only the terms of the first order over the electric field and we will obtain the partial differential cross-sections of SB in the case of linear theory. Thus, we can obtain the asymptotic formula for the partial differential cross-sections of SB process in the weak wave field $\mathbf{A}(t)$ :

$$
\begin{aligned}
\frac{d \Lambda^{(\text {linear })}}{d \theta}=\frac{2 m_{*}^{2}}{\pi \hbar^{3}\left|\mathbf{p}_{0}\right|} \mid \widetilde{U} & {\left.\left[\frac{\mathbf{p}-\mathbf{p}_{0}}{\hbar}\right]\right|^{2} \mid\left(1+e^{\left.i 2 \zeta\left[\vartheta\left(\mathbf{p}_{0}\right)-\vartheta(\mathbf{p})\right)\right]}\right) \delta_{n 0} \delta_{\mathcal{E} \mathcal{E}_{0}} } \\
& +\left[\frac{\chi}{2 i} C_{1} \delta_{n-1}\right] \delta_{\mathcal{E}\left(\mathcal{E}_{0}+\hbar \omega\right)} \\
& -\left.\left[\frac{\chi}{2 i} C_{1} \delta_{n+1}\right] \delta_{\mathcal{E}\left(\mathcal{E}_{0}-\hbar \omega\right)}\right|^{2} d \mathcal{E} .
\end{aligned}
$$

Here the time dependent $C_{1}$ coefficient is defined as:

$$
\begin{gathered}
C_{1}=-\left(\frac{p_{x}}{\sqrt{m_{*} \hbar \omega}}-\frac{p_{0 x}}{\sqrt{m_{*} \hbar \omega}}\right)\left(1+e^{\left.i 2 \zeta\left[\vartheta\left(\mathbf{p}_{0}\right)-\vartheta(\mathbf{p})\right)\right]}\right) \\
+2 \zeta \frac{\left(\mathcal{E}-\mathcal{E}_{0}\right)}{\hbar \omega}\left[\frac{\sqrt{m_{*} \hbar \omega} p_{y}}{p^{2}}-\frac{\sqrt{m_{*} \hbar \omega} p_{0 y}}{p_{0}^{2}}\right] e^{i 2 \zeta\left[\vartheta\left(\mathbf{p}_{0}\right)-\vartheta(\mathbf{p})\right]} .
\end{gathered}
$$

Comparing the $n$-photon cross-section $d \Lambda^{(n)}$ (18) of SB process with the elastic one, we conclude that formula (18) at $\mathbf{A}(\tau)=0(n=0)$ passes to elastic scattering cross-section $d \Lambda_{\text {elast }}$ [16], which is the analog of the Mott formula but in $2 \mathrm{D}$ scattering theory:

$$
\begin{gathered}
\frac{d \Lambda_{\text {elast }}}{d \theta}=\frac{2 m_{*}^{2}}{\pi \hbar^{3}\left|\mathbf{p}_{0}\right|}\left|\widetilde{U}\left[\frac{\mathbf{p}-\mathbf{p}_{0}}{\hbar}\right]\right|^{2} \\
\times\left|\left(1+e^{i 2 \zeta\left[\vartheta\left(\mathbf{p}_{0}\right)-\vartheta(\mathbf{p})\right]}\right)\right|^{2}
\end{gathered}
$$

The phase term

$$
\left(1+\exp i 2 \zeta\left[\vartheta\left(\mathbf{p}_{0}+\frac{e}{c} \mathbf{A}(\tau)-\vartheta\left(\mathbf{p}+\frac{e}{c} \mathbf{A}(\tau)\right)\right]\right)^{2}\right.
$$

in Eq. (18) at $\mathbf{A}(\tau)=0$ is the known overlap factor

$$
\left(1+\sigma e^{-i \theta_{q}}\right)\left(1+\sigma^{\prime} e^{i \theta_{q}}\right)=2\left(1+\sigma \sigma^{\prime} \cos \theta_{q}\right),
$$

where $\theta_{q}=2 \zeta \vartheta\left(\mathbf{p}_{0}\right)-2 \zeta \vartheta(\mathbf{p})$. The term (22) is a wave function form-factor associated with the chiral nature of BG, and the last is determined by its band structure. 

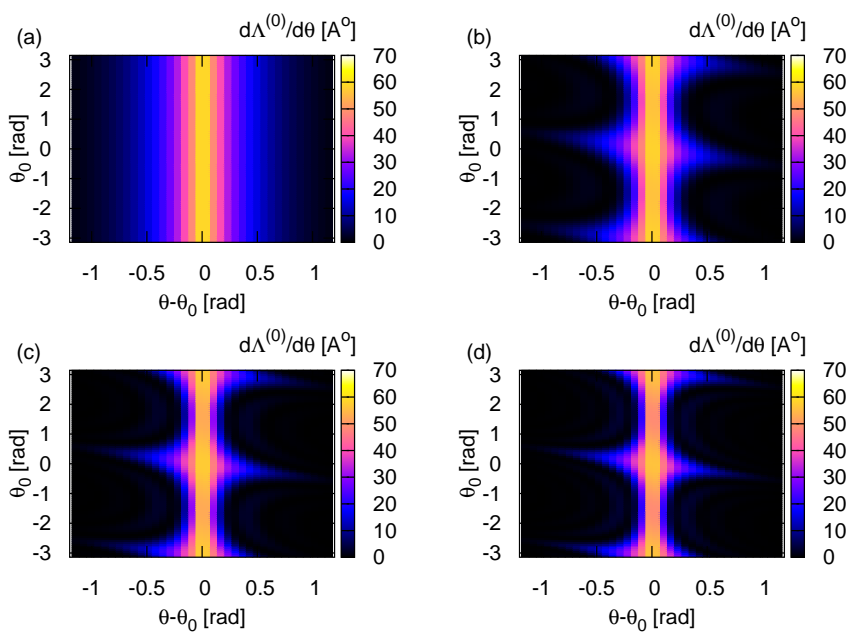

FIG. 1: (Color online) Partial differential cross section $d \Lambda^{(n)} / d \theta$ (in angstrom) of SB process for photon number $n=0$ vs the electron deflection angle $\theta-\theta_{0}$ and incident angle $\theta_{0}$ for linear polarization of EM wave of intensities: (a) $\chi=0$, (b) $\chi=1$, (c) $\chi=1.5$, and (d) $\chi=2$.

\section{CROSS-SECTIONS OF STIMULATED BREMSSTRAHLUNG ON THE SCREENING COULOMB POTENTIAL OF IMPURITY IONS IN DOPED BILAYER GRAPHENE}

Bellow we will obtain the partial differential cross-sections in particular case of SB process on a screened Coulomb potential of impurity ions in BG, using Eq. (18). In an analytic form of Coulomb screening in BG [23], [58 60] has been performed in analogous with the case of intrinsic SG [41], [44]. So, the Fourier transform $\widetilde{U}(\mathbf{q})=\int U(\mathbf{r}) e^{-i \mathbf{q r}} d \mathbf{r}$ of a charged impurity center of potential can be written as:

$$
\widetilde{U}(\mathbf{q})=\frac{2 \pi e^{2}}{\kappa q \epsilon(q)}
$$

where the screening term $\epsilon(q)(q=|\mathbf{q}|)$ is the 2D finite temperature static dielectric function in random phase approximation (RPA) appropriate for BG, given by the formula [23], [59]:

$$
\epsilon(q)=1+\frac{q_{s}}{q}\left[g(q)-f(q) \Theta\left(q-2 k_{F}\right)\right] .
$$

Here $k_{F}=\sqrt{2 m_{*} \varepsilon_{F}} / \hbar$ is $2 \mathrm{D}$ Fermi wave vector in BG case, $q_{s}=k_{T F} / k_{F}=4 m_{*} e^{2} \log 4 /\left(\kappa \hbar^{2}\right)\left(\sim n^{-1 / 2}\right.$ for BG [59]) is the 2D Thomas-Fermi screening wave vector given by $k_{T F}[58]$ scaled on $k_{F}$, which controls the dimensionless strength of quantum screening; and $\kappa$ is the background lattice dielectric constant of the system. The function $\Theta\left(q-2 k_{F}\right)$ is the step function (or staircase function). The functions $g(q), f(q)$ are defined by the formulas [59]:

$$
\begin{gathered}
g(q)=\frac{1}{2} \sqrt{4+\bar{q}^{4}}-\log \left[\frac{1+\sqrt{1+\bar{q}^{4} / 4}}{2}\right], \\
f(q)=\left(1+\frac{\bar{q}^{2}}{2}\right) \sqrt{1-\frac{4}{\bar{q}^{2}}}+\log \left[\frac{\bar{q}-\sqrt{\bar{q}^{2}-4}}{\bar{q}+\sqrt{\bar{q}^{2}-4}}\right],
\end{gathered}
$$

where $\bar{q}=q / k_{F}$. This usual 2D dispersion or the static screening [23] one is the particular case of the wave vector dependent plasmon dispersion and the wave frequency dependent screening function case [61]. For the last dielectric function and screening show very different behavior than in usual 2D graphene case, and the plasmons creation will be actual.

For the following numerical considerations it is convenient to represent the differential cross-sections of SB on the charged impurities in the form of dimensionless quantities. We will obtain the final form for the dimensionless partial differential cross-sections of SB process $d \Lambda^{(n)} / d \theta$, taking into account Eqs. (9), (18), and (23) with relations Eqs. (24)(26), in the certain case of linearly polarized EM wave with the dimensionless vector potential $\overline{\mathbf{A}}(t)=-\widehat{\mathbf{e}} \chi \sin (2 \pi \tau)$ 

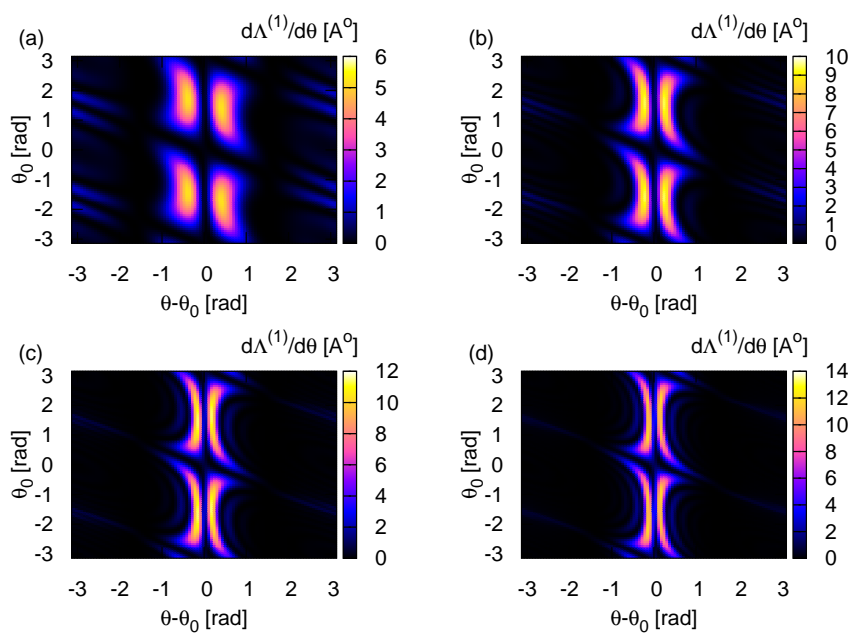

FIG. 2: (Color online) Partial differential cross section $d \Lambda^{(n)} / d \theta$ (in angstrom) of SB process for photon number $n=1$ and laser intensities: (a) $\chi=0.5$, (b) $\chi=1$, (c) $\chi=1.5$, and (d) $\chi=2$.

scaled on the wavelength $\lambda=2 \pi c / \omega$ (unit vector $\widehat{\mathbf{e}}$ is directed along the axis $O X$ ):

$$
\begin{gathered}
\frac{d \Lambda^{(n)}}{\lambda d \theta}=\frac{1}{2400 \log ^{2} 4} \frac{1}{\bar{\varepsilon}_{0}} \frac{\left|\overline{\mathbf{p}}_{0}\right|}{\overline{m_{*} \mathrm{~V}_{F}}} \\
\times \frac{\overline{\hbar q}_{s}^{2}}{\left|\overline{\mathbf{p}}-\overline{\mathbf{p}}_{0}\right|^{2} \epsilon^{2}\left(\left|\overline{\mathbf{p}}-\overline{\mathbf{p}}_{0}\right|\right)} \\
\times \mid \int_{0}^{1} d \tau\left(1+e^{i 2 \zeta\left[\vartheta\left(\overline{\mathbf{p}}_{0}-\chi \sin (2 \pi \tau)\right)-\vartheta(\overline{\mathbf{p}}-\chi \sin (2 \pi \tau))\right]}\right) \\
\times \exp \left\{i 2 \pi n \tau-\pi i \int_{0}^{\tau}\left[\left(\left(\bar{p}_{x}-\chi \sin \left(2 \pi \tau^{\prime}\right)\right)^{2}+\bar{p}_{y}^{2}-2 \overline{\mathcal{E}}\right)\right.\right. \\
\left.\left.-\left(\left(\bar{p}_{0 x}-\chi \sin \left(2 \pi \tau^{\prime}\right)\right)^{2}+\bar{p}_{0 y}^{2}-2 \overline{\mathcal{E}}_{0}\right)\right] d \tau^{\prime}\right\}\left.\right|^{2} \\
\times \delta\left(\overline{\mathcal{E}}-\overline{\mathcal{E}}_{0}-n\right) d \overline{\mathcal{E}} .
\end{gathered}
$$

In Eq. (27) the dimensionless momentum, energy, 2D screening vector, 2D Fermi velocity, and time are defined as follows:

$$
\begin{gathered}
\bar{p}_{x, y}=\frac{p_{x, y}}{\sqrt{m_{*} \hbar \omega}}, \overline{\mathcal{E}}=\frac{\mathcal{E}}{\hbar \omega}, \\
\overline{\hbar q}_{s}=\frac{\hbar q_{s}}{\sqrt{m_{*} \hbar \omega}}, \overline{m_{*} \mathrm{~V}_{F}}=\frac{m_{*} \mathrm{v}_{F}}{\sqrt{m_{*} \hbar \omega}}, d \tau=\frac{d t}{T} .
\end{gathered}
$$

As mentioned in Introduction, the intensity $I_{\chi}$ strongly depends on the parameter $\chi$ and the photon energy. At $\chi \gtrsim 1$ the multiphoton effects become essential. So, for numerical analysis of multiphoton SB cross sections in graphene we assume coherent EM radiation with intensity dimensionless parameter $\chi \gtrsim 1$ in particular case of the fixed photon energy $\hbar \omega=0.01 \mathrm{eV}\left(\lambda=1.24 \times 10^{6} \AA\right)$ from terahertz domain. The Fermi energy is taken to be $\varepsilon_{F}=20 \hbar \omega\left(\varepsilon_{F} \gg \hbar \omega\right)$, dielectric environment constant is $\kappa=2.5$ for an impurity strength in the presence of the $\mathrm{SiO}_{2}$ 

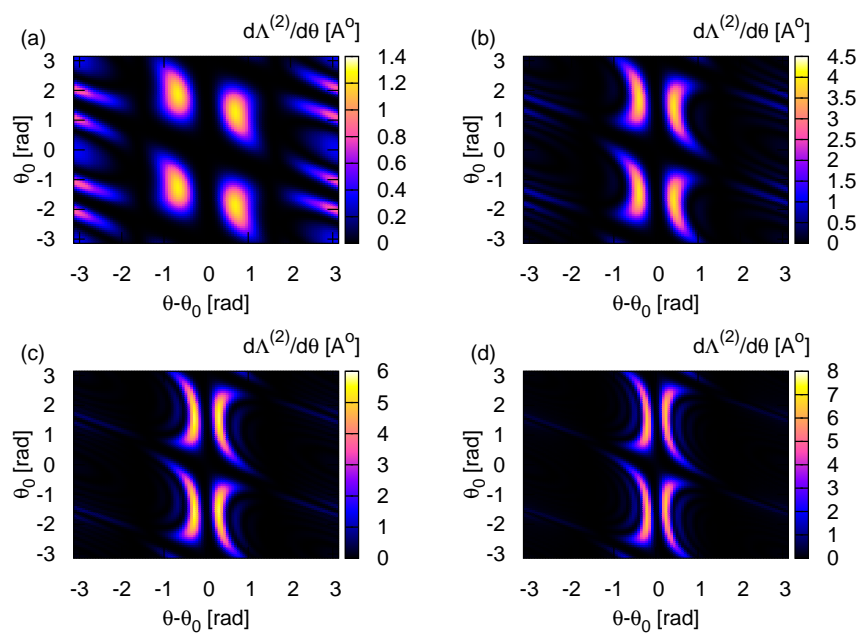

FIG. 3: (Color online) Partial differential cross section $d \Lambda^{(n)} / d \theta$ (in angstrom) for photon number $n=2$ : $(a)-(d)$ correspond to dimensionless field parameters $\chi=0.5,1,1.5$ and 2 , respectively.
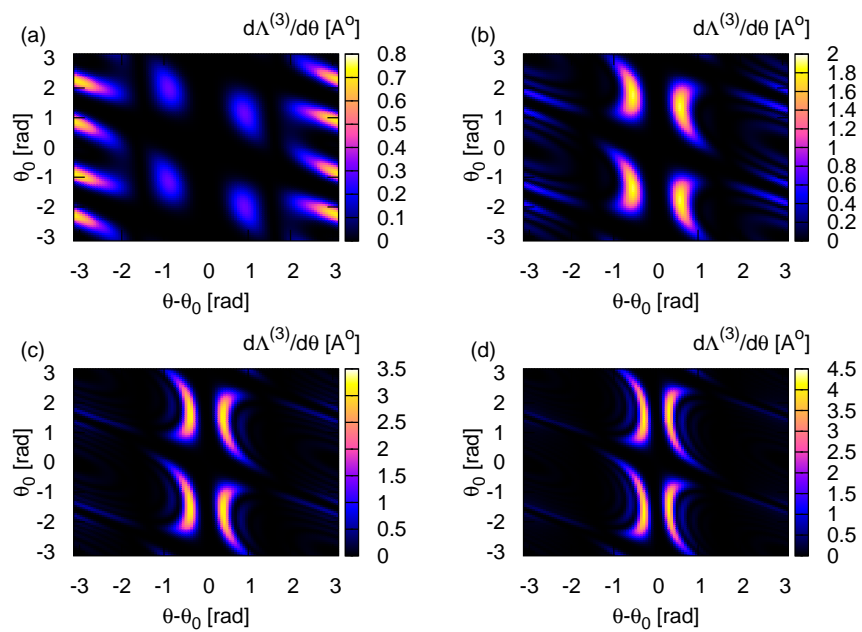

FIG. 4: (Color online) Partial differential cross section $d \Lambda^{(n)} / d \theta$ (in angstrom) for photon number $n=3$ : $(a)-(d)$ correspond to dimensionless field parameters $\chi=0.5,1,1.5$ and 2 , respectively.

substrate [30]. Note, that while for graphene on $\mathrm{SiO}_{2}$ substrate corresponding to $\kappa=2.5$ [44], in particularly, covering $\mathrm{BG}$ on $\mathrm{SiO}_{2}$ with ice, it can be change the last to $\kappa=3.5$, which would increase the conductivity by $10 \%$ for permitted charged impurity density $\sim 10^{12} \mathrm{~cm}^{-2}[30$. The band dispersion is quadratic and only the lowest subband is occupied [17, 21, 23], and the RPA theory applies in the density domain $10^{10} \mathrm{~cm}^{-2}<n<5 \times 10^{12} \mathrm{~cm}^{-2}$. In particular case for a homogeneous carrier density $n=10^{11} \mathrm{~cm}^{-2}$, the dimensionless effective screening vector $q_{s}$ defined by the formula [59]:

$$
q_{s}=\frac{54.8}{\sqrt{n \times 10^{-10} \mathrm{~cm}^{2}}},
$$

we have taken $q_{s}=17.329$. In the context of graphene, it has given useful direct comparison between screening in SG versus screening in BG:

$$
q_{T F}^{B G} / q_{T F}^{S G} \approx 16 / \sqrt{n \times 10^{-10} \mathrm{~cm}^{2}}
$$

which showing that as carrier density decreases, BG screening becomes much stronger than SG screening [59]. The quantum dynamics of multiphoton emission and absorption processes at the electron-impuarity ion SB is studied making time integration in Eq. (27) by the fourth-order Runge-Kutta method. The all numerical results are for the valley $\zeta=1$. The figures in the following illustrate the SB at different intensities of stimulated wave of linear 

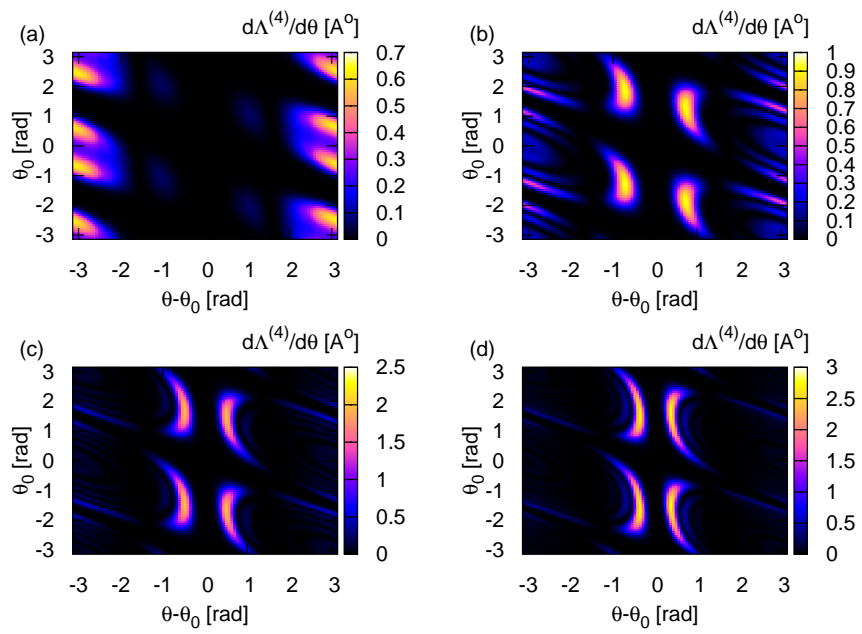

FIG. 5: (Color online) Partial differential cross section $d \Lambda^{(n)} / d \theta$ (in angstrom) for photon number $n=4$ : $(a)-(d)$ correspond to dimensionless field parameters $\chi=0.5,1,1.5$ and 2 , respectively.

polarization. To reveal the peculiarities which can be associated with the chiral nature of BG and its parabolic dispersion, the comparison with the SG case has been made.

In the Figs. 1-5, the envelopes of partial differential cross sections $d \Lambda^{(n)} / d \theta$ (27) of SB in BG are plotted as a function of the electron deflection angle $\theta-\theta_{0}$ and the angle $\theta_{0}$ between the vector of the electron initial momentum and electric field strengths of an coherent EM radiation field. Then, Fig. 1 shows the elastic scattering part $(n=0)$, and Figs. 2-5 correspond to SB for different number of absorbed photons $n>0$. Similar calculations for emitted photons at $n<0$ show qualitatively the same picture, so that it is omitted. The angular dependence of partial differential cross sections in these figures are displayed for various intensities: in Fig. 1 (a) at $\chi=0$, (b) $\chi=1$ $\left(I_{\chi}=6 \times 10^{4} \mathrm{Wcm}^{-2}\right)$, (c) $\chi=1.5\left(I_{\chi}=1.35 \times 10^{5} \mathrm{Wcm}^{-2}\right)$, and (d) $\chi=2\left(I_{\chi}=2.4 \times 10^{5} \mathrm{Wcm}^{-2}\right)$, and in Figs. 2-5: (a) $\chi=0.5\left(I_{\chi}=1.5 \times 10^{4} \mathrm{Wcm}^{-2}\right)$, (b) $\chi=1$, (c) $\chi=1.5$, and (d) $\chi=2$. As is seen, the maximum values of the multiphoton SB partial cross sections at $n=0$ are reached in the direction of the electric field at $\theta-\theta_{0} \simeq 0$. But with the increase of the photon number and the wave intensity the angular distribution becomes more asymmetric. In comparison with the SG case one can note, that maximum values of the multiphoton cross sections correspond substantially different values of the deflection angle $\theta-\theta_{0}$. Particularly, with the increase of the photon number, the multiphoton cross section maximums at a weak wave intensity $\chi=0.5$ (closed to main elastic result) are reached at deflection angles $\theta-\theta_{0}= \pm \pi$. This demonstrates that the probability of the scattering under large angles (backscattering) is considerable. As is seen from theses figures, with the increase of the photon number and the wave intensity, the SB partial differential cross sections' maximums are shifted to the direction of the wave electric field.

To demonstrate the dependence of SB cross sections on the number of emitted or absorbed photons, the envelopes of the partial cross sections $n \Lambda^{(n)}$ for various angle $\theta_{0}$ between the initial electron momentum and the wave electric field, are presented in Fig.6. For this goal, the SB partial differential cross section (27) is integrated over the scattering angle of the outgoing electron for fixed initial angle $\theta_{0}$ and at various pump wave intensities. For terahertz photons, the multiphoton interaction regime in BG can be achieved already at the intensities $I_{\chi} \sim 10^{4} \mathrm{Wcm}^{-2}$. Figure 6 shows that with the increase of coherent radiation intensity, the multiphoton cut-off number increases; and, compared to the one-photon scattering in linear theory (19), the multiphoton effect becomes dominant. As one can see, at considered for numerical calculations intensities multiphoton SB process have strongly nonlinear behavior. Hence, one can expect strong deviation for the wave absorption in doped bilayer graphene from linear case. Note, that in Fig. 6 the doped BG partial cross-sections behavior is the similar to the intrinsic SG one for the certain initial angle $\theta_{0}=\pi / 2 \mathrm{rad}$ only. As is seen from Fig. 6, the envelopes of the partial cross sections $n \Lambda^{(n)}$ for $n>1(n<1)$ have many characteristic maxima (minima) in the doped BG case which is in sharp contrast with the doped SG case [50] where for the same interaction parameters one has a single maximum (minimum) in absorption (emission) part. Next, we see from Fig. 6 that with the increase of radiation intensity the absorption becomes dominant over emission process. Thus, the SB absorption process in doped BG can be feasible already at moderate radiation intensities.

The total partial cross sections $\Lambda^{(n)}$ for the elastic, one, and two photon processes versus the intensity for various initial angles $\theta_{0}$ are presented in Fig. 7. As is seen from this figure, if the elastic cross section $\Lambda^{(0)}$ decreases with the increase of induced radiation intensity, the partial cross sections $\Lambda^{(1)}$ and $\Lambda^{(2)}$ grow with the increase of intensity. In the presence of strong radiation field the elastic cross section is essentially modified. Hence, the strong coherent 


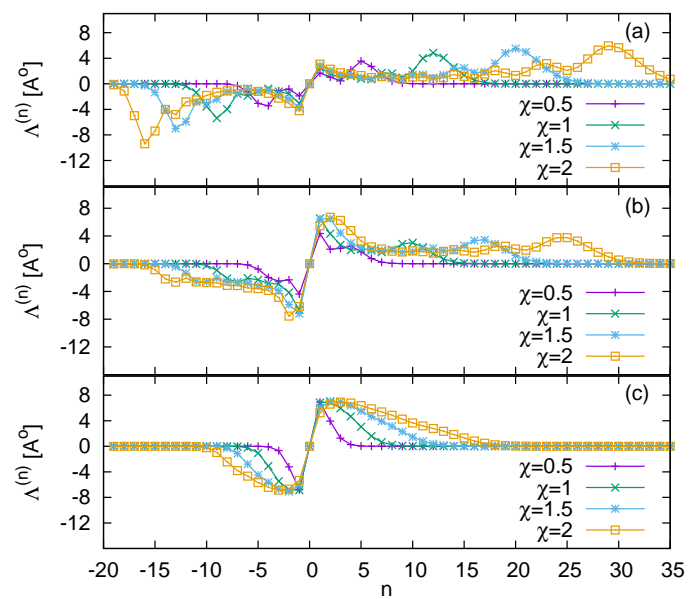

FIG. 6: (Color online) Envelopes of partial absorption-emission cross sections $n \Lambda^{(n)}$ (in angstrom) as a function of the number of emitted or absorbed photons. The angle $\theta_{0}$ between the initial electron momentum and wave electric field is taken: (a) 0 , (b) $\pi / 4 \mathrm{rad}$, and (c) $\pi / 2 \mathrm{rad}$.

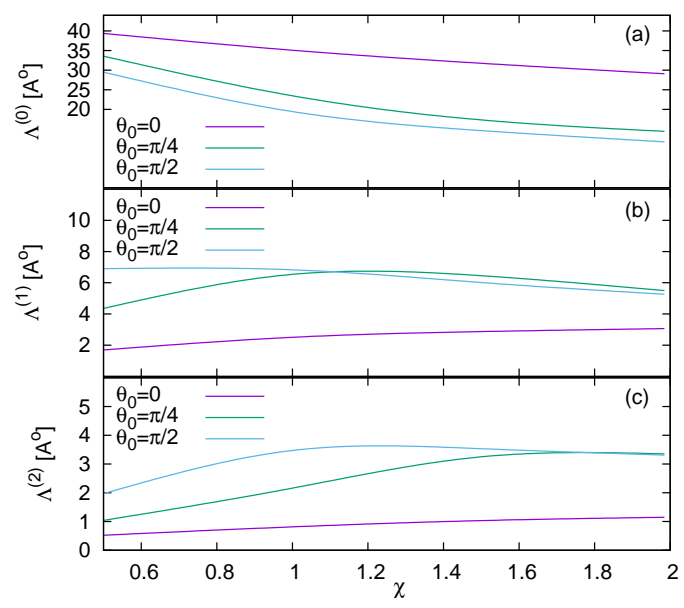

FIG. 7: (Color online) Partial cross sections $\Lambda^{(n)}$ (in angstrom) versus intensity parameter for several initial angle $\theta_{0}$ between the electron momentum and wave electric field. Photon number is taken: (a) $n=0$ (elastic case), (b) $n=1$, and (c) $n=2$.

radiation field can allow to manipulate with the electron transport properties of doped BG. Moreover, as is seen from Figs. 1-6 and comparison with the results for SG case [50], such procedure can be more effective in BG case.

\section{CONCLUSION}

The theoretical investigation of the multiphoton SB process in doped BG is given. Based on the quantum theory, we study the induced scattering of 2D chiral particles on the charged impurity ions of an arbitrary electrostatic potential in the Born approximation. To exclude the interband transitions in doped BG, we have considered high Fermi energies and an external EM wave is taken to be in the terahertz domain of frequencies. The obtained analytical formulas for SB in coherent radiation field with linear polarization are analyzed numerically for a screened Coulomb potential of doped into BG impurity ions. The latter shows that induced by a power radiation field the scattering process in BG is strongly nonlinear. In comparison with the SG case the new behavior has been demonstrated, which may be associated with the chiral nature of BG and its parabolic energy dispersion. In particular, it has been obtained many maxima of SB partial absorption cross-section in doped BG at multiphoton excitation in moderately intense radiation fields. In addition, the elastic cross section is significantly modified by the pump wave, which opens new possibilities to manipulate the wave dressed electron transport properties in doped BG. The comparison with the 
results of intrinsic SG case demonstrates that absorption of a strong electromagnetic radiation in doped BG is of special interest, and this problem will be addressed in the forthcoming article.

\section{Acknowledgments}

The authors are deeply grateful to prof. H. K. Avetissian for permanent discussions and valuable recommendations. This work was supported by the RA MES State Committee of Science.

[1] K. S. Novoselov, A. K. Geim, S. V. Morozov, D. Jiang, Y. Zhang, S. V. Dubonos, I. V. Grigorieva, and A. A. Firsov, "Electric field effect in atomically thin carbon films", Science 306(5696), 666-669 (2004), http://dx.doi.org/10.1126/science.1102896

[2] A. H. Castro Neto, F. Guinea, N. M. R. Peres, K. S. Novoselov, and A. K. Geim, "The electronic properties of graphene", Rev. Mod. Phys. 81(1), 109-162 (2009), http://dx.doi.org/10.1103/RevModPhys.81.109.

[3] A. K. Geim, "Graphene: Status and prospects", Science 324(5934), 1530-1534 (2009), http://dx.doi.org/10.1126/science.1158877.

[4] K. S. Novoselov, A. K. Geim, S. V. Morozov, D. Jiang, M. I. Katsnelson, I. V. Grigorieva, S. V. Dubonos, and A. A. Firsov, "Two-dimensional gas of massless Dirac fermions in graphene", Nature 438, 197-200 (2005), http://dx.doi.org/10.1038/nature04233

[5] H. K. Avetissian, "Relativistic Nonlinear Electrodynamics: The QED Vacuum and Matter in Super-Strong Radiation Fields", monograph: 978-3-319-26384-7, Springer, New York, 2016.

[6] H. K. Avetissian, A. K. Avetissian, G. F. Mkrtchian, Kh. V. Sedrakian, "Creation of particle-hole superposition states in graphene at multiphoton resonant excitation by laser radiation", Phys. Rev. B 85(11), 115443(1)-115443(10) (2012), http://dx.doi.org/10.1103/PhysRevB.85.115443.

[7] H. K. Avetissian, A. G. Ghazaryan, G. F. Mkrtchian, "Relativistic theory of inverse-bremsstrahlung absorption of ultrastrong laser radiation in plasma", J. Phys. B 46, 205701(1)-205701(9) (2013), http://dx.doi.org/10.1088/0953-4075/46/20/205701.

[8] H. K. Avetissian, A. G. Ghazaryan, H. H. Matevosyan, G. F. Mkrtchian, "Microscopic nonlinear relativistic quantum theory of absorption of powerful x-ray radiation in plasma", Phys. Rev. E 92, 043103(1)-043103(8) (2015), http://dx.doi.org/10.1103/PhysRevE.92.043103

[9] H. K. Avetissian, A. K. Avetissian, G. F. Mkrtchian, Kh. V. Sedrakian, "Multiphoton resonant excitation of FermiDirac sea in graphene at the interaction with strong laser fields", J. Nanophoton 6, 061702(1)-061702(17) (2012), http://dx.doi.org/10.1117/1.JNP.6.061702

[10] H. K. Avetissian, G. F. Mkrtchian, K. G. Batrakov, S. A. Maksimenko, A. Hoffmann, "Nonlinear theory of graphene interaction with strong laser radiation beyond the Dirac cone approximation: Coherent control of quantum states in nano-optics", Phys. Rev. B 88, 245411(1)-245411(7) (2013), http://dx.doi.org/10.1103/PhysRevB.88.245411.

[11] H. K. Avetissian, G. F. Mkrtchian, K. G. Batrakov, S. A. Maksimenko, A. Hoffmann, "Multiphoton resonant excitations and high-harmonic generation in bilayer graphene", Phys. Rev. B. 88, 165411(1)-165411(9) (2013), http://dx.doi.org/10.1103/PhysRevB.88.165411.

[12] H. K. Avetissian, A. G. Ghazaryan, G. F. Mkrtchian, and Kh. V. Sedrakian, "High harmonic generation in Landauquantized graphene subjected to a strong electromagnetic radiation", J. Nanophoton 11, 016004(1)-016004(9) (2017), http://dx.doi.org/10.1117/1.JNP.11.016004

[13] H. K. Avetissian, G. F. Mkrtchian, "Coherent nonlinear optical response of graphene in the quantum Hall regime", Phys. Rev. B 94(4), 045419(1)-045419(7) (2016), http://dx.doi.org/10.1103/PhysRevB.94.045419

[14] K. Xu, S. Liu, W. Sun, Zh. Ma, Z. Li, Qi Yu, and G. Li, "Design and fabrication of a monolithic optoelectronic integrated Si CMOS LED based on hot-carrier effect", IEEE Journal of Selected Topics in Quantum Electronics 22, Issue: 6, Nov.-Dec. (2016), http://dx.doi.org/10.1109/JSTQE.2016.2517980

[15] E. McCann and V. I. Fal'ko, "Landau-level degeneracy and quantum Hall effect in a graphite bilayer", Phys. Rev. Lett. 96, 086805(1)-086805(4) (2006), https://doi.org/10.1103/PhysRevLett.96.086805.

[16] M. Koshino and T. Ando, "Transport in bilayer graphene: calculations within a self-consistent Born approximation", Phys. Rev. B 73, 245403(1)-245403(8) (2006), https://doi.org/10.1103/PhysRevB.73.245403.

[17] I. Snyman and C.W.J. Beenakker, "Ballistic transmission through a graphene bilayer", Phys. Rev. B 75, 045322(1)045322(6) (2007), https://doi.org/10.1103/PhysRevB.75.045322.

[18] K. Novoselov, E. McCann, S. V. Morozov, V. I. Fal'ko, M. I. Katsnelson, U. Zeitler, D. Jiang, F. Schedin, and A. K. Geim, "Unconventional quantum Hall effect and Berry's phase of 2pi in bilayer graphene", Nature Phys. 2, 177-180 (2006), https://doi.org/10.1038/nphys245.

[19] S. Das Sarma, A. K. Geim, P. Kim, and A. H. MacDonald Recent, "Exploring graphene: research advances", A special issue of Solid State Communications 143(1-2), pp. 1-122 Elsevier, New York, 2007.

[20] H. Liu, Y. Li, Y. S. You, Sh. Ghimire, T. F. Heinz, and D. A. Reis, "High-harmonic generation from an atomically thin semiconductor", Nature Physics 13, 262-265 (2017), https://doi.org/10.1038/nphys3946. 
[21] S. Morozov, K. Novoselov, M. Katsnelson, F. Schedin, D. Elias, J. Jaszczak, and A. Geim, "Giant intrinsic carrier mobilities in graphene and its bilayer", Phys. Rev. Lett. 100, 016602(1)-016602(4) (2008), https://doi.org/10.1103/PhysRevLett.100.016602.

[22] J. Oostinga, H. B. Heersche, X. Liu, A. F. Morpurgo, L. M. Vandersypen, "Gate-induced insulating state in bilayer graphene devices", Nature Mater. 7, 151-157 (2008), http://dx.doi.org/10.1038/nmat2082.

[23] E. H. Hwang and S. Das Sarma, "Screening, Kohn anomaly, Friedel oscillation, and RKKY interaction in bilayer graphene", Phys. Rev. Lett. 101, 156802(1)-156802(4) (2008), http://dx.doi.org/10.1103/PhysRevLett.101.156802

[24] F. Hipolito, A. Taghizadeh, and T. Garm Pedersen, "Nonlinear optical response of doped mono- and bilayer graphene: Length gauge tight-binding model", Phys. Rev. B. 98, 205420(1)-205420(11) (2018), http://dx.doi.org/10.1103/PhysRevB.98.205420.

[25] E. McCann, "Asymmetry gap in the electronic band structure of bilayer graphene", Phys. Rev. B 74, 161403(1)-161403(6) (2006), https://doi/10.1103/PhysRevB.74.161403.

[26] Y. Zhang, T.-T. Tang, C. Girit, Zh. Hao, M. C. Martin, A. Zettl, M. F. Crommie, Y. R. Shen, and F. Wang, "Direct observation of a widely tunable band gap in bilayer graphene", Nature 459, 820-823 (2009), https://doi.org/10.1038/nature08105.

[27] K. F. Mak, Ch. H. Lui, J. Shan, and T. F. Heinz, "Observation of an electric-field-induced band gap in bilayer graphene by infrared spectroscopy", Phys. Rev. Lett. 102, 256405(1)-256405(4) (2009), https://doi.org/10.1103/PhysRevLett.102.256405.

[28] J. Nilsson, A. H. Castro Neto, F. Guinea, and N. M. R. Peres, "Electronic properties of graphene multilayers", Phys. Rev. Lett. 97, 266801(1)-266801(4) (2006), https://doi.org/10.1103/PhysRevLett.97.266801.

[29] J. Nilsson, A. H. Castro Neto, F. Guinea, and N. M. R. Peres, "Electronic properties of bilayer and multilayer graphene", Phys. Rev. B 78, 045405(1)-045405(7) (2008), https://doi.org/10.1103/PhysRevB.78.045405.

[30] S. Das Sarma, E. H. Hwang, and E. Rossi, "Theory of carrier transport in bilayer graphene", Phys. Rev. B 81, 161407R(1)161407(4)R (2010), http://dx.doi.org/10.1103/PhysRevB.81.161407.

[31] H. K. Avetissian, A. K. Avetissian, A. G. Ghazaryan, Kh. V. Sedrakian, and G. F. Mkrtchian, "High-order harmonic generation in gapped bilayer graphene", preprint arXiv:1905.08016, https://arxiv.org/abs/1905.08016.

[32] A. K. Avetissian, A. G. Ghazaryan, Kh. V. Sedrakian, "Third harmonic generation in gapped bilayer graphene", preprint arXiv:1905.08189, https://arxiv.org/abs/1905.08189.

[33] F. V. Bunkin, A. E. Kazakov, M. V. Fedorov, "Interaction of intense optical radiation with free electrons (nonrelativistic case)", Sov. Phys.-Usp. 15, 416-435 (1973), http://iopscience.iop.org/0038-5670/15/4/R04.

[34] M. H. Mittleman, "Introduction to the theory of laser-atom interactions", Plenum, New York, 1993.

[35] B. Dóra and R. Moessner, "Nonlinear electric transport in graphene: quantum quench dynamics and the Schwinger mechanism", Phys. Rev. B. 81(16), 165431(1)-165431(6) (2010), http://dx.doi.org/10.1103/PhysRevB.81.165431.

[36] M. M. Denisov, M. V. Fedorov, "Bremsstrahlung effect on relativistic electrons in a strong radiation field", Sov. Phys. JETP 26, 779-783 (1968), http:// jetp.ac.ru/cgi-bin/dn/e_022_04_0844.pdf.

[37] T. R. Hovhannisyan, A. G. Markossian, G. F. Mkrtchian, "On the theory of the relativistic cross-sections for stimulated bremsstrahlung on an arbitrary electrostatic potential in the strong electromagnetic field", Eur. Phys. J. D 20, 17-25 (2002), http://dx.doi.org/10.1140/epjd/e2002-00110-7.

[38] T. Ando, "Screening effect and impurity scattering in monolayer graphene", J. Phys. Soc. Jpn. 75, 074716(1)-074716(7) (2006), http://dx.doi.org/10.1143/JPSJ.75.074716

[39] J.-H. Chen, C. Jang, S. Adam, M. S. Fuhrer, E. D. Williams, and D.M. Ishigami, "Charged-impurity scattering in graphene", Nature Physics 4, 377-381 (2008), http://dx.doi.org/10.1038/nphys935

[40] K. Nomura and A. H. MacDonald, "Quantum Hall ferromagnetism in graphene", Phys. Rev. Lett. 96, 256602(1)-256602(4) (2006), http://dx.doi.org/10.1103/PhysRevLett.96.256602.

[41] E. H. Hwang, S. Adam, and S. Das Sarma, "Carrier transport in two-dimensional graphene layers", Phys. Rev. Lett. 98, 186806(1)-186806(4) (2007), http://dx.doi.org/10.1103/PhysRevLett.98.186806.

[42] D. S. Novikov, "Elastic scattering theory and transport in graphene", Phys. Rev. B 76, 245435(1)-245435(17) (2007), http://dx.doi.org/10.1103/PhysRevB.76.245435.

[43] Y.-W. Tan, Y. Zhang, K. Bolotin, Y. Zhao, S. Adam, E.H. Hwang, S. Das Sarma, H. L. Stormer, and P. Kim, "Measurement of scattering rate and minimum conductivity in graphene", Phys. Rev. Lett. 99, 246803(1)-246803(4) (2007), http://dx.doi.org/10.1103/PhysRevLett.99.246803

[44] M. I. Katsnelson, "Nonlinear screening of charge impurities in graphene", Phys. Rev. B. 74, 201401(R)(1)-201401(R)(3) (2006), http://dx.doi.org/10.1103/PhysRevB.74.201401.

[45] D. P. DiVincenzo and E. J. Mele, "Self-consistent effective-mass theory for intralayer screening in graphite intercalation compounds", Phys. Rev. B 29, 1685-1694 (1984), http://dx.doi.org/10.1103/PhysRevB.29.1685.

[46] N. H. Shon and T. Ando, "Quantum transport in two-dimensional graphite system", J. Phys. Soc. Jpn. 67, 2421-2429 (1998), http://dx.doi.org/10.1143/JPSJ.67.2421

[47] H. Suzuura and T. Ando, "Crossover from symplectic to orthogonal class in a two-dimensional honeycomb lattice", Phys. Rev. Lett. 89, 266603(1)-266603(4) (2002), http://dx.doi.org/10.1103/PhysRevLett.89.266603.

[48] N. M. R. Peres, F. Guinea, and A. H. Castro Neto, "Electronic properties of disordered two-dimensional carbon", Phys. Rev. B 73, 125411(1)-125411(23) (2006), http://dx.doi.org/10.1103/PhysRevB.73.125411.

[49] S. Sun and J.-L. Zhu, "Impurity spectra of graphene under electric and magnetic fields", Phys. Rev. B 89, 155403(1)155403(10) (2014), http://dx.doi.org/10.1103/PhysRevB.89.155403

[50] A. K. Avetissian, A.G. Ghazaryan, K. V. Sedrakian, and B. R. Avchyan, "Induced nonlinear cross sections of conductive electrons scattering on the charged impurities in doped graphene", J. Nanophoton. 11, 036004(1)-036004(11) (2017), 
https://doi.org/10.1117/1.JNP.11.036004.

[51] A. K. Avetissian, A.G. Ghazaryan, K. V. Sedrakian, and B. R. Avchyan, "Microscopic nonlinear quantum theory of absorption of strong EM radiation in doped graphene", J. Nanophoton. 12, 016006(1)-016006(12) (2018), https://doi.org/10.1117/1.JNP.12.016006.

[52] S. Adam and S. Das Sarma, "Boltzmann transport and residual conductivity in bilayer graphene", Phys. Rev. B 77, 115436(1)-115436(6) (2008), https://doi.org/10.1103/PhysRevB.77.115436.

[53] M. I. Katsnelson, "Scattering of charge carriers by point defects in bilayer graphene", Phys. Rev. B 76, 073411(1)-073411(3) (2007), https://doi.org/10.1103/PhysRevB.76.073411.

[54] M. Monteverde, C. Ojeda-Aristizabal, R. Weil, K. Bennaceur, M. Ferrier, S. Gueron, C. Glattli, H. Bouchiat, J.N. Fuchs, D.Maslov, "Transport and elastic scattering times as probes of the nature of impurity scattering in single and bilayer graphene", Phys. Rev. Lett. 104, 126801(1)-126801(4) (2010), http://dx.doi.org/10.1103/PhysRevLett.104.126801.

[55] W. Zhang and L.-J. Li, "The screening of charged impurities in bilayer graphene", New Journal of Physics 12, 103037(1)103037(6) (2010), http://dx.doi.org/10.1088/1367-2630/12/10/103037.

[56] D. K. Patel, A. C. Sharma, "Electron-impurity scattering rate in doped bilayer graphene", AIP Conference Proceedings 1447, 973-976 (2012), https://doi.org/10.1063/1.4710329.

[57] Sh. Xiao, J.-H. Chen, Sh. Adam, E. D. Williams, and M. S. Fuhrer, "Charged impurity scattering in bilayer graphene", Phys. Rev. B 82, 041406(1)-041406(4) (2010), https://doi.org/10.1103/PhysRevB.82.041406.

[58] T. Ando, A.B. Fowler, F. Stern, "Electronic properties of two-dimensional systems", Rev. Mod. Phys. 54, 437-672 (1982), http://dx.doi.org/10.1103/RevModPhys.54.437

[59] S. D. Sarma, Sh. Adam, E. H. Hwang, and E. Rossi, "Electronic transport in two-dimensional graphene", Rev. Mod. Phys. 83, 407-415 (2011), http://dx.doi.org/10.1103/RevModPhys.83.407

[60] K. Xu, L. W. Snyman, H. Aharoni, "Si light-emitting device in integrated photonic CMOS ICs", Optical Materials 69, 274-282 (2017), http://dx.doi.org/10.1016/j.optmat.2017.03.055

[61] E. H. Hwang and S. Das Sarma, "Dielectric function, screening, and plasmons in two-dimensional graphene ", Phys. Rev. B. 75, 205418(1)-205418(6) (2007), https://doi.org/10.1103/PhysRevB.75.205418. 